\title{
Effect of sodium aescinate treatment on PCOS rat model with insulin resistance
}

\author{
Chen $\mathrm{L}^{1}$, Hu $\mathrm{LM}^{1}$, Wang $\mathrm{YF}^{1}$, Yang $\mathrm{HY}^{1}$, Huang $\mathrm{XY}^{1}$, Zhou $\mathrm{W}^{1}$, Sun $\mathrm{HX}^{2}$ \\ Reproductive Medicine Center, Changzhou Maternal and Child Health Care Hospital, the affiliated hospital \\ of Nanjing Medical University, Changzhou, Jiangsu, China. Haixiangdt@outlook.com
}

\begin{abstract}
BACKGROUND: Recent studies indicated that insulin resistance may contribute to the pathogenesis of polycystic ovary syndrome (PCOS); however, the specific mechanism is still unclear.

OBJECTIVE: To investigate the effect of sodium aescinate (SA) on PCOS-IR rat models.

METHODS: Sixty rats were randomly divided into the five groups: un-treated rats $(n=12)$, PCOS-IR group $(\mathrm{n}=12)$, PCOS-IR group plus $50 \mathrm{mg} / \mathrm{kg} \mathrm{SA}(\mathrm{n}=12)$, PCOS-IR group plus $10 \mathrm{mg} / \mathrm{kg} \mathrm{SA}(\mathrm{n}=12)$, PCOS-IR group plus $150 \mathrm{mg} / \mathrm{kg}$ metformin $(n=12)$. On day 21, rats were sacrificed, and H\&E staining was performed for histopathologic examination of the ovaries; moreover, the serum level of follicle-stimulating hormone (FSH), testosterone, and luteotropic hormone (LH) were measured, and the expression as well as phosphorylation of $\mathrm{PI} 3 \mathrm{~K}$, Akt and Gsk-3ß were examined using western blot assay.

RESULTS: High dosage of SA treatment improved the morphological features of the ovaries in PCOS rats, and also induced significant decrease in serum expression of testosterone and LH/FSH ratio and significant decrease in the expression of p-PI3K, p-Akt and p-Gsk-3ß.

CONCLUSION: Our results demonstrated that SA treatment could alleviate the symptom of PCOS in rat model through regulating the PI3K/Akt/GSK3- $\beta$ pathway (Fig. 4, Ref. 22). Text in PDF www.elis.sk.

KEY WORDS: sodium aescinate, polycystic ovary syndrome, insulin resistance, PI3K, AKT, GSK3- $\beta$.
\end{abstract}

\section{Introduction}

Polycystic ovary syndrome (PCOS) is a common female endocrine disorder characterized by hyperandrogenism, ovulatory dysfunction and polycystic ovaries (1). It is highly prevalent among women at the reproductive age (about $7 \%$ ). In addition to reproductive consequences, like anovulation and infertility, patients with PCOS also exhibit metabolic abnormalities i.e. obesity, insulin resistance (2), metabolic syndrome, hyperinsulinemia, dyslipidemia and nonalcoholic steatohepatitis (NASH) (3). The etiology of PCOS remains unclear, and most of the patients with PCOS (approximately 50-70\%) experience the varying degrees of IR, suggesting that the occurrence of IR may play important roles in the pathogenesis of PCOS. In clinical application, metformin based therapies have been considered as the most common anti-PCOS treatments, however, long-term metformin treatment may lead to side effects e.g. diarrhea, gastrointestinal upset (4). Thus, at cur-

${ }^{1}$ Reproductive Medicine Center, Changzhou Maternal and Child Health Care Hospital, the affiliated hospital of Nanjing Medical University, Changzhou, Jiangsu, China, and ${ }^{2}$ Reproductive Medicine Center, Nanjing Drum Tower Hospital, Nanjing, Jiangsu, China

Address for correspondence: H.X. Sun, Reproductive Medicine Center, Drum Tower Hospital, No. 321 Zhongshan Road, Gulou District, Najing, Jiangsu 210008, China.

Phone: +86.13915838896 .

Acknowledgement: This research study was financially supported by Applied Basic Research Programs of Science Technology of Changzhou City (CJ20140021). rent stage, there is no cure for PCOS, and great efforts still need to be made to identify novel effective medication for treating PCOS.

Natural plant extracts have been applied for many years in the healthcare industry, either as dietary supplements or medications, for their excellent therapeutic properties and low adverse effects (5). Sodium aescinate (SA) is a triterpene saponin that is extracted from the seeds of chestnut (Aesculus hippocastanum), and the therapeutic properties of SA (e.g. anti-oxidant, anti-inflammatory, relieving tissue edema and recovering vasopermeability) have been discussed in many previous works (6-9). There are different formulations of SA in clinical applications, such as injections, oral tablets and topical gels. Up to now, the function of SA as an anti-PCOS drug has not yet been investigated. In the present study, we explored the effect of SA in PCOS rat models. We hypothesized that SA could attenuate the symptoms of PCOS and improve PCOS-related abnormalities through regulating the PI3K/Akt/GSK-3 $\beta$ pathway.

\section{Materials and methods}

\section{Animals}

Sixty female Sprague-Dawley (SD) rats $(250 \pm 50$ g) at $8-9$ weeks of age with normal estrous cycles were used in this study. All rats were housed in a $22^{\circ} \mathrm{C}$ room with a 12 -h light/dark cycle and provided with standard diet and water. This study was approved by the Animal Studies Committee of Nanjing Medical University. All animals received humane care in compliance with the criteria published by the national institutes of health (NIH publication 86-23, revised 1985). 


\section{3-227}

\section{Treatments}

The rats were randomly divided into the five groups (12 animals per group): the healthy Sprague-Dawley rats that received only dimethyl sulfoxide (DMSO; solvent of letrozole) that served as the control group; SD rats received a letrozole-induced PCOS(PCOS model group); letrozole-induced PCOS rats treated with $50 \mathrm{mg} / \mathrm{kg}$ SA (SA high dosage group); letrozole-induced PCOS rats treated with $10 \mathrm{mg} / \mathrm{kg} \mathrm{SA}$ (SA medium dosage group); letrozole-induced $\mathrm{PCOS}$ rats received $150 \mathrm{mg} / \mathrm{kg}$ metformin (positive control group). The PCOS was induced by oral gavage of letrozole (dissolved in DMSO) once daily at a concentration of $1 \mathrm{mg} / \mathrm{kg}$ for 21 consecutive days as Di Pietro et al (2) described. Mice were sacrificed on day 21 , and the ovaries were collected for future analysis.

\section{Histopathological evaluation}

Ovaries of the rats in each group were fixed in $10 \%$ neutral formalin for $24 \mathrm{~h}$. The ovaries were then sectioned into $4-\mu \mathrm{m}$ and stained with hematoxylin and eosin. Then the histological feature of every sample was observed and analyzed using an Olympus BX51 microscope (Olympus, Tokyo, Japan).

\section{Biochemical analysis}

Insulin resistance was measured according to the homeostasis model assessment for insulin resistance (HOMA-IR)(10). Briefly, the venous blood of the rats was extracted 12 hours after fasting, and the fasting blood glucose (FBG) and fasting insulin (FINS) were examined using commercially available kits (Nanjing Jiancheng Bioengineering Institute, Nanjing, China). The degree of the HOMA-IR was quantified as HOMA-IR=FBG (mmol/L)FINS (mU/L)/22.5, and HOMA-IR > 2.8 was considered as insulin resistant. Serum levels of follicle-stimulating hormone (FSH), testosterone, and luteotropic hormone ( $\mathrm{LH}$ ) were measured using competitive enzyme-linked immunosorbent assay (ELISA) methods with commercially available ELISA kits (Nanjing Jiancheng Bioengineering Institute, Nanjing, China).

The extraction of total RNA and real-time reverse transcriptionquantitative polymerase chain reaction (RT-qPCR)

Total RNAs were extracted from the ovary samples using Trizol reagent (Invitrogen, Thermo Fisher Scientific, Waltham, MA, USA) according the manufacturer's instructions. Then cDNAs were synthesized using Prime Script RT reagent kit (Takara, Dalian, China) and RT-PCR was performed using the SYBR ExScript RT-PCR kit (TaKaRa, Dalian, China) on an ABI 7300 Real-Time PCR System (Applied Biosystems, Foster City, CA, USA). The thermocycling profile was: $95^{\circ} \mathrm{C}$ for $30 \mathrm{sec}$; followed by 40 cycles of denaturation at $95^{\circ} \mathrm{C}$ for $3 \mathrm{sec}$ and annealing at $60{ }^{\circ} \mathrm{C}$ for $30 \mathrm{sec}$. The relative expression of the genes in each sample was normalized to the level of GAPDH using the $2^{-\Delta \Delta \mathrm{Ct}}$ method. The sequence of the primes were: GAPDH, Forward 5'-CATCAGCAATGCCTCCTGCAC-3', Reverse 5'-TGAGTCCTTCCACGATACCAAAGTT-3'; PI3K, Forward 5'-CTGTGTGGGACTTATTGAGGTGGT-3', Reverse 5'-ACTGATGTAGTGTGTGGCTGTTGA-3'; AKT, Forward 5'-TGTGAAGGAGGGTTGGCTGC-3', Reverse 5'-ACTGCGCCACAGAGAAGTTGTT-3'; GSK3 $\beta$, Forward
5'-GACGCTCCCTGTGATtTATGTC-3', Reverse 5'-GTTAGTCGGGCAGTTGGTGTAT-3'.

\section{Western blot}

Proteins were extracted from ovary samples using radioimmunoprecipitation assay (RIPA) buffer (Beyotime, Shanghai, China), and the concentration of the proteins were determined using Pierce ${ }^{\mathrm{TM}}$ BCA Protein Assay Kit (Thermo Fisher Scientific, Waltham, MA, USA). Then proteins were separated by electrophoresis, and transferred onto topolyvinylidene fluoride membranes; next, the membranes were blocked with 5\% non-fat milk in tris-buffered saline and incubated with primary antibodies (rat anti-PI3K, anti-p-PI3K, anti-Akt, anti-p-Akt, anti-Gsk-3 $\beta$, antip-Gsk-3 $\beta$ and anti- $\beta$-actin; diluted at 1:2000, all purchased from Santa Cruz) at $4{ }^{\circ} \mathrm{C}$. Then the membranes were incubated with HRP conjugated secondary antibody (1:20,000, Santa Cruz) for $1.5 \mathrm{~h}$ at room temperature. The protein bands were visualized using ChemiDoc ${ }^{\mathrm{TM}} \mathrm{XRS}+$ imaging system (Bio-Rad, Hercules, CA, USA).

\section{Statistics}

The experimental continuous data are shown as the mean \pm standard deviation. A one-way analysis of variance (ANOVA) was used to the distribution of data. In all statistical analyses, $p<0.05$ was recognized as statistically significant. We conducted our statistical analyses with the SPSS 17.0 (SPSS Inc., Chicago, IL).

\section{Results}

Effect of SA on insulin resistance in PCOS rat models

Of the 60 rats, 36 exhibited PCOS with insulin resistance (HOMA-IR > 2.8), and the value of HOMA-IR of the rats were shown in Figure 1. The HOMA-IR value of the model group was significantly higher than the control group, and the HOMA-IR values of the SA and metformin groups were markedly lower than in the model group (Fig. 1).

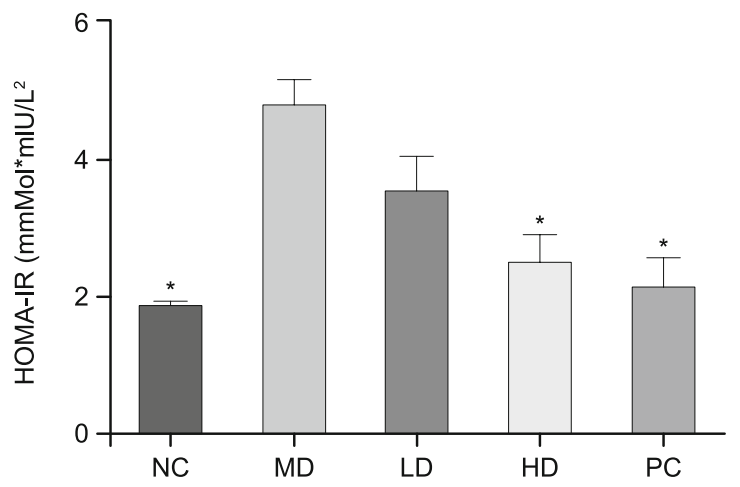

Fig. 1. Values of homeostasis model assessment of insulin resistance (HOMA-IR) in different groups. * $\mathrm{p}<0.05$ compared to MD group. HOMA-IR - homeostasis model assessment of insulin resistance; NC - the un-treated control group; MD - PCOS-IR model group; LD low dosage SA group; HD - high dosage SA group; PC - metformin treated group. 

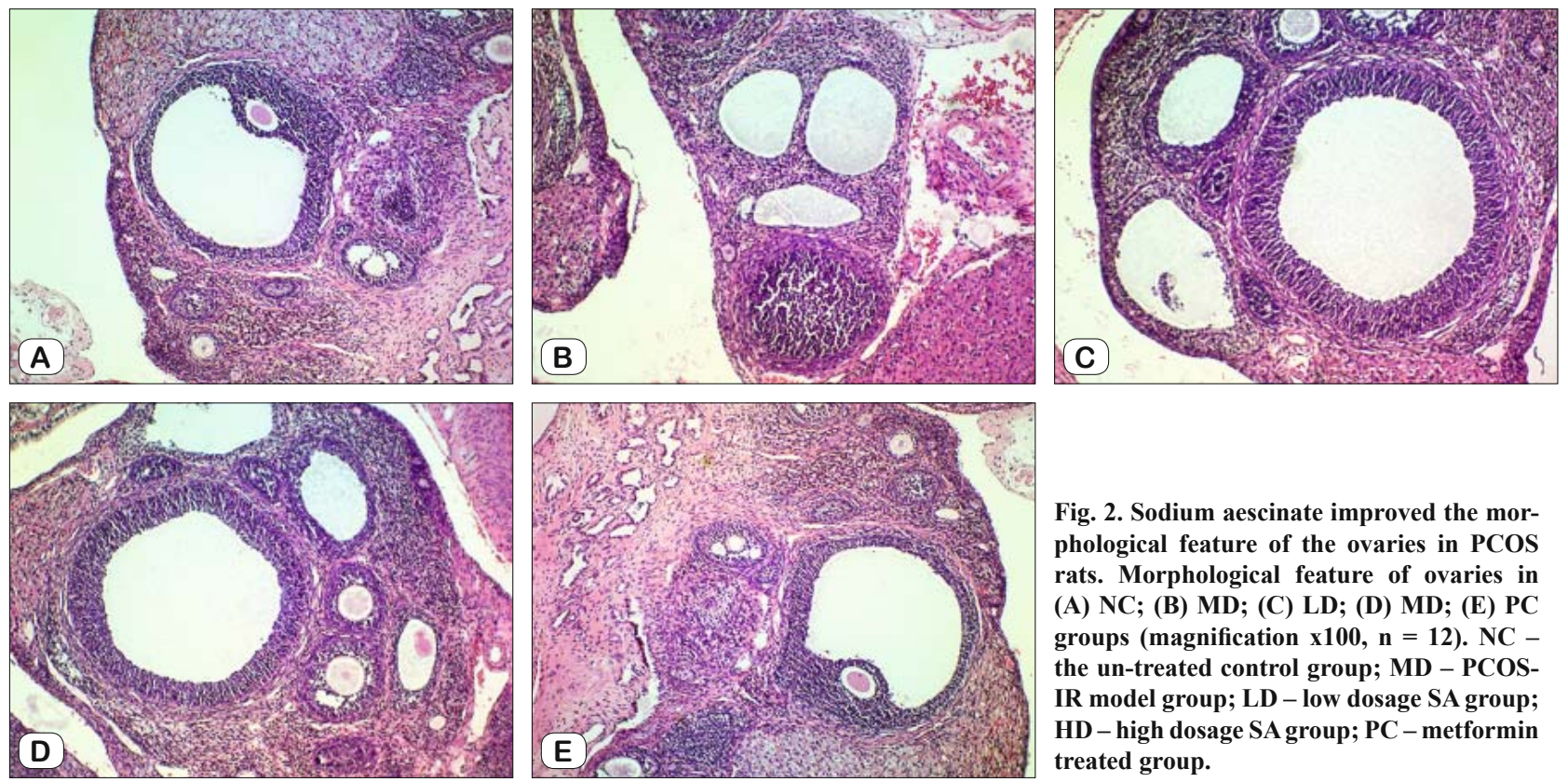

Fig. 2. Sodium aescinate improved the morphological feature of the ovaries in PCOS rats. Morphological feature of ovaries in (A) NC; (B) MD; (C) LD; (D) MD; (E) PC groups (magnification $x 100, n=12$ ). $N C-$ the un-treated control group; MD - PCOSIR model group; LD - low dosage SA group; HD - high dosage SA group; PC - metformin treated group.
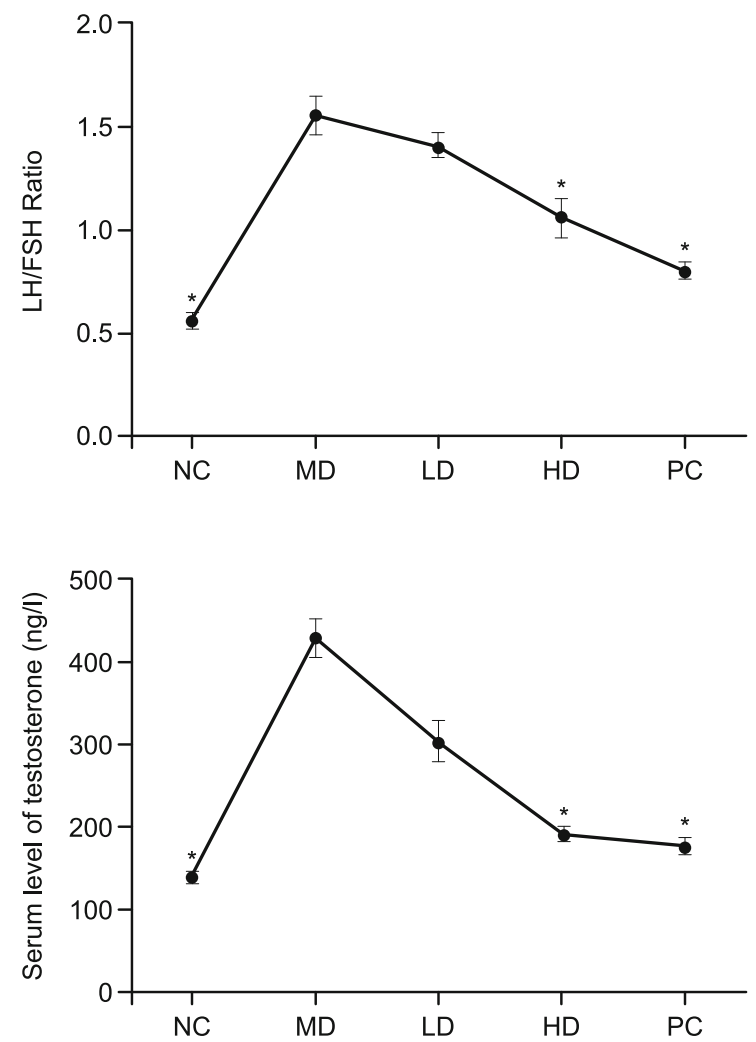

Fig. 3. Effect of sodium aescinate treatment on serum LH/FSH ratio and levels of testosterone in PCOS rat models. (A) LH/FSH ratio in different groups; (B) Serum level of testosterone in different groups. * $\mathbf{p}<0.05$ compared to MD group. $\mathrm{LH}$ - luteotropic hormone; FSH - follicle-stimulating hormone; NC - the un-treated control; MD PCOS-IR model group; LD - low dosage SA group; HD - high dosage SA group; PC - metformin treated group.

Morphological features of the rat ovarian in different groups

On day 21 , mice were sacrificed, and the morphological features of the ovaries in the different groups were observed to evaluate the therapeutic effect of SA on the PCOS rats. As shown in the Figure 2, the ovaries of rats in the control group exhibited identical clinical feature, with follicles in different stages and 8-9 layers of granulosa cells in the dominant follicle (Fig. 2A); on the other hand, the ovaries of rats in the model group showed cystic dilatation of the follicles, in which the oocytes were absent, and there were only 3-4 layers of granulosa cells in the follicle (Fig. 2B). In high dosage of SA (Fig. 2D) and metformin (Fig. $2 \mathrm{E})$ treated groups, the $\mathrm{H} \& \mathrm{E}$ staining results of the ovaries have revealed follicles at different stages and increased layers (3-7) of granulosa cells in the follicle compared to the ovaries from the model group (Fig. 2).

Effect of SA treatment on serum levels of testosterone and LH/ FSH ratio in PCOS rat models

The serum levels of testosterone and LH/FSH ratio in each group were shown in the Figure 3. Compared to the control group, there was a significant increase in the expression of testosterone and LH/FSH ratio in the PCOS rat models $(\mathrm{p}<0.05)$, meanwhile SA and metformin treated PCOS rats showed a marked reduction in the serum level of testosterone and the ratio of LH/FSH. Among the medication treated groups, metformin and high dosage of SA showed the most-pronounced decrease in the serum level of testosterone and the ratio of LH/FSH $(\mathrm{p}<0.05)$.

Effect of SA on the expression of PI3K/Akt/GSK-3 $\beta$ in ovaries of PCOS rats

To further investigate the therapeutic effect of SA in PCOS rats, we examined the expression and phosphorylation of PI3K, 


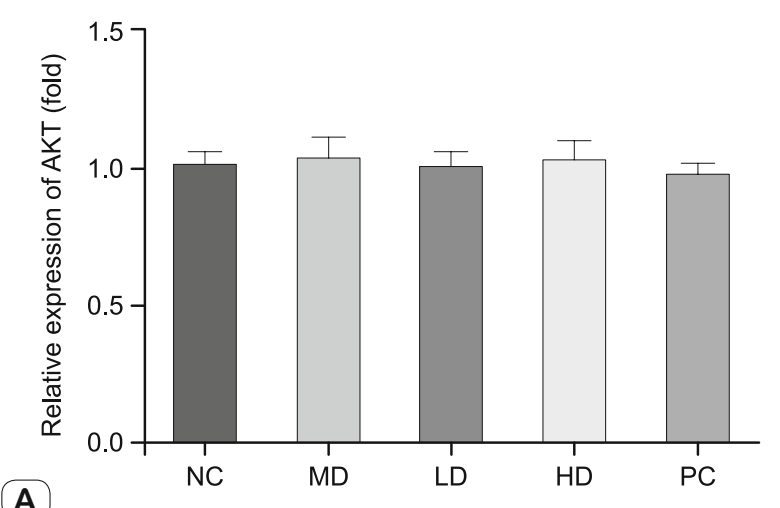

A

B
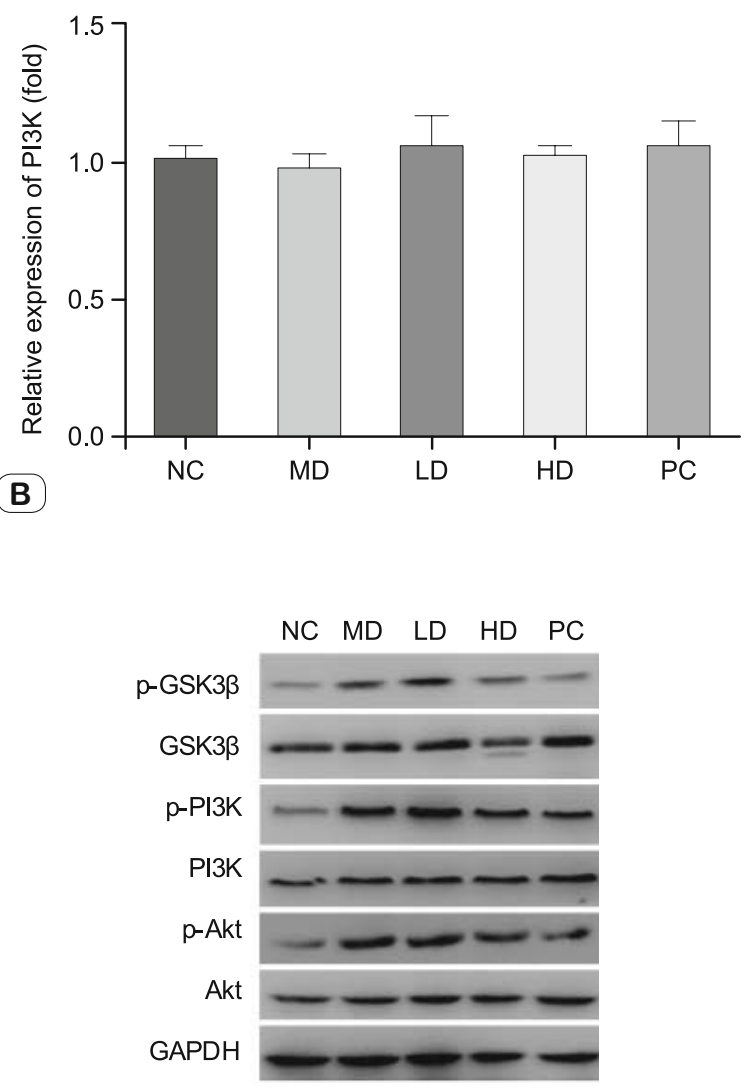

(D)

(C)

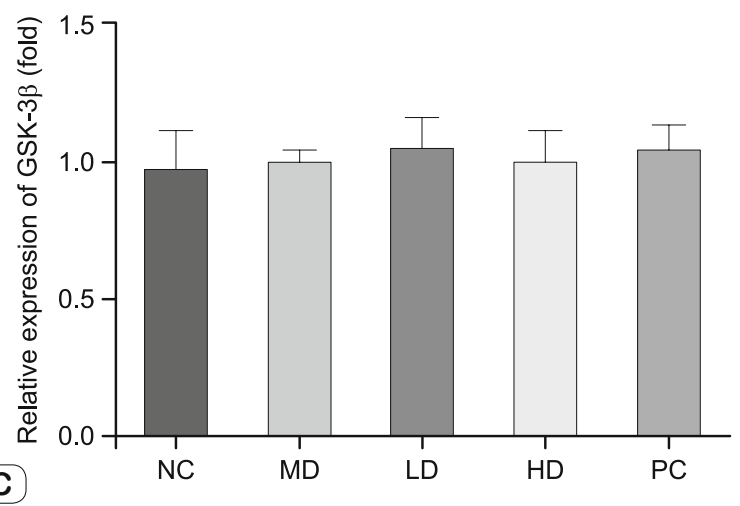

Fig. 4. Expression of PI3K, Akt and GSK3- $\beta$ in different groups (A) Relative mRNA expression of PI3K in different groups; (B) Relative mRNA expression of Akt in different groups; (C) Relative mRNA expression of GSK3- $\beta$ in different groups; (D) Relative protein expression of PI3K, pPI3K, Akt, p-Akt, GSK3- $\beta$ and p-GSK3- $\beta$ in different groups. * $p<0.05$ compared to MD group. NC - the un-treated control; MD - PCOS-IR model group; LD - low dosage SA group; HD - high dosage SA group; PC - metformin treated group; PI3K - phosphatidylinositol 3-kinase; p-PI3K - phosphorylated PI3K; Akt - protein kinase B; p-Akt - phosphorylated Akt; GSK3- $\beta$ - Glycogen synthase kinase 3-Beta; p-GSK3- $\beta$ - phosphorylated GSK3- $\beta$; GAPDH - glyceraldehyde-3-phosphate dehydrogenase.

Akt, GSK-3 $\beta$ in the ovaries of PCOS rats using western blotting methods, and the Figure 4 shows the results. While the expression of PI3K, Akt, GSK-3 $\beta$ showed no significant differences among each group on both RNA and protein level, the phosphorylation of PI3K, Akt, GSK-3 $\beta$ was significantly increased in the model group compared to the control group (Fig. 4D); moreover, the phosphorylation of PI3K, Akt, GSK-3 $\beta$ was markedly decreased in the SA high dosage and metformin treated PCOS rats (Fig. 4D).

\section{Discussion}

The therapeutic roles of SA in different diseases have been discussed previously. Zhang et al established diabetic rat models, and proved that SA can effectively improve the process of wound healing in rat diabetic models (11); Du et al proved that SA may play a liver protective role in methyl parathion induced rat liver injury models (6); Wei (9) et al discovered that SA may alleviate the symptoms of acute lung injury rats through modulating the expression of MMP-9, SOD and MDA; Cheng et al proved that SA may have neuroprotection role in traumatic spinal cord injury rat models by reducing the oxidative stress(12). In the present study, we successfully established PCOS rat model, and proved that in the ovaries of high dosage of SA treated PCOS rats, the follicles were at different stages and have increased layers of granulosa cells in the follicle compared to the ovaries from the model group, suggesting that high dosage of SA treatment can improve the morphological features and alleviate the morbid condition of ovaries in PCOS rats.

The pathogenesis of PCOS is still unclear. Majority of the experts in this field believed that IR is an important factor in the occurrence and progress of PCOS (13). A high incidence of IR has been demonstrated in patients with PCOS. Previous studies indicated that insulin might increase the secretion of LH and Gonadotropin-releasing hormone $(\mathrm{GnRH})$ in PCOS (14); moreover, insulin may also effect the expression of the low-density lipoprotein cholesterol receptors (LDLRs) in granulosa cells, which can further induce the synthesis of testosterone in theca cells (15). Then, an increased expression of testosterone can block the sensitivity of GnRH to progesterone and estradiol, leading to reduced progesterone negative feedback and the development of 
PCOS (16). In the present study, we discovered that SA treatment not only improved the morphological features of the ovaries, but also improves the IR in PCOS rats, as indicated by the decrease in the HOMA-IR value in the high dosage of SA treated group compared to the model group; moreover, there was a significant reduction in the serum level of testosterone and the ratio of $\mathrm{LH} /$ FSH in metformin and SA groups compared to the PCOS group $(\mathrm{p}<0.05)$. Taken together, these results indicated that SA might improve insulin resistant of the PCOS rats.

The roles of phosphatidylinositol 3-kinase (PI3K)/protein kinase B (Akt) pathway in glucose and insulin metabolism have been proved in many previous studies (17). In PCOS, the PI3K/Akt pathway may be involved in many cellular and molecular activity i.e. cell proliferation, differentiation, apoptosis, transformation and movement; moreover, PI3K/Akt signaling pathway may also contribute to the insulin resistance, obesity, endocrine disorders and abnormal follicular development in PCOS(18). Glycogen synthase kinase 3 (GSK3) is a serine /threonine protein kinase, and GSK3- $\beta$, one isoform of GSK3, has been proved to participate in the process neuron development (19), energy metabolism (20), and the formation of the body patterns (21). In PCOS, it has been reported that the tyrosine phosphorylation of GSK3- $\beta$ was enhanced, and insulin-stimulated serine phosphorylation of GSK3- $\beta$ was reduced (22). In our study, we observed that either high dosage of SA or metformin treatment had no influence on the expression of PI3K, AKT and GSK3- $\beta$ on both mRNA and protein level ( $p>0.05)$; however, high dosage of SA and metformin treatment induced a significant decrease in the phosphorylation of PI3K, AKT and GSK3- $\beta$. These data indicated that SA could reverse IR through regulating the phosphorylation of the PI3K/ Akt/GSK3- $\beta$ signaling pathway.

Our study had some limitations. We only performed animal studies, however, a simple animal model may not completely reproduce a complex human disease; thus, in the following works, we should also confirm our results using human cell lines, and also perform clinical studies to verify the therapeutic efficacy of SA on PCOS patients.

To sum up, our results demonstrated that SA treatment could alleviate the symptoms of PCOS in rat model through regulating the PI3K/AKT/GSK3- $\beta$ pathway, indicating that SA has the potential to become an effective medication for treating PCOS.

\section{References}

1. Mohamadin AM, Habib FA, Al-Saggaf AA. Cardiovascular disease markers in women with polycystic ovary syndrome with emphasis on asymmetric dimethylarginine and homocysteine. Ann Saudi Med 201; 30: 278-283.

2. Di Pietro M, Parborell F, Irusta G et al.: Metformin regulates ovarian angiogenesis and follicular development in a female polycystic ovary syndrome rat model. Endocrinology 2015; 156: 1453-1463.

3. Weerakiet S. Polycystic ovary syndrome and the metabolic syndrome. J Med Assoc Thai 2004; 87 (Suppl 3): S189-193.

4. Aghahosseini M, Aleyaseen A, Safdarian L, Moddaress-Hashemi S, Mofid B, Kashani L. Metformin 2,500 mg/day in the treatment of obese women with polycystic ovary syndrome and its effect on weight, hormones, and lipid profile. Arch Gynecol Obstet 2010; 282: 691-694.
5. Sinha P, Srivastava S, Mishra N, Yadav NP. New perspectives on antiacne plant drugs: contribution to modern therapeutics. Biomed Res Int 2014; 301-304.

6. Du Y, Wang T, Jiang $\mathbf{N}$ et al. Sodium aescinate ameliorates liver injury induced by methyl parathion in rats. Exp Ther Med 2012; 3: 818-822.

7. Du Y, Wang T, Jiang $\mathbf{N}$ et al. Protective effect of sodium aescinate on lung injury induced by methyl parathion. Hum Exp Toxicol 2011; 30: 1584-1591.

8. Wang YK, Han J, Xiong WJ et al. Evaluation of in vivo antioxidant and immunity enhancing activities of sodium aescinate injection liquid. Molecules 2012; 17: 10267-10275.

9. Wei T, Tong W, Wen-ping $\mathrm{S}$ et al. The impact of sodium aescinate on acute lung injury induced by oleic acid in rats. Exp Lung Res 2011; 37: 585-599.

10. Keskin M, Kurtoglu S, Kendirci M, Atabek ME, Yazici C. Homeostasis model assessment is more reliable than the fasting glucose/insulin ratio and quantitative insulin sensitivity check index for assessing insulin resistance among obese children and adolescents. Pediatrics 2005; 115: e500-503.

11. Zhang Z, Cao G, Sha L, Wang D, Liu M. The Efficacy of Sodium Aescinate on Cutaneous Wound Healing in Diabetic Rats. Inflammation 2015; 38: 1942-1948.

12. Cheng P, Kuang F, Ju G. Aescin reduces oxidative stress and provides neuroprotection in experimental traumatic spinal cord injury. Free Radic Biol Med 2016; ;99: 405-417.

13. Sun X, Wu X, Zhou Y, Yu X, Zhang W. Evaluation of Apelin and Insulin Resistance in Patients with PCOS and Therapeutic Effect of Drospirenone-Ethinylestradiol Plus Metformin. Med Sci Monit 2015; 21:2547-2552.

14. Rojas J, Chavez M, Olivar L et al. Polycystic ovary syndrome, insulin resistance, and obesity: navigating the pathophysiologic labyrinth. Int J Reprod Med 2014; 2014: 719050.

15. Blank SK, McCartney CR, Chhabra S et al. Modulation of gonadotropin-releasing hormone pulse generator sensitivity to progesterone inhibition in hyperandrogenic adolescent girls - implications for regulation of pubertal maturation. J Clin Endocrinol Metab 2009; 94: 2360-2366.

16. Padmanabhan V, Veiga-Lopez A. Sheep models of polycystic ovary syndrome phenotype. Mol Cell Endocrinol 2013; 373: 8-20.

17. Schultze SM, Hemmings BA, Niessen M, Tschopp O. PI3K/AKT, MAPK and AMPK signalling: protein kinases in glucose homeostasis. Expert Rev Mol Med 2012; 14: e1.

18. Makker A, Goel MM, Das V, Agarwal A. PI3K-Akt-mTOR and MAPK signaling pathways in polycystic ovarian syndrome, uterine leiomyomas and endometriosis: an update. Gynecol Endocrinol 2012; 28: 175-181.

19. Salcedo-Tello P, Ortiz-Matamoros A, Arias C. GSK3 Function in the Brain during Development, Neuronal Plasticity, and Neurodegeneration. Int J Alzheimers Dis 2011; 189728.

20. Wang Y, Feng W, Xue W et al. Inactivation of GSK-3beta by metallothionein prevents diabetes-related changes in cardiac energy metabolism, inflammation, nitrosative damage, and remodeling. Diabetes 2009; 58: 1391-1402.

21. Chen H, Fajol A, Hoene M et al. PI3K-resistant GSK3 controls adiponectin formation and protects from metabolic syndrome. Proc Natl Acad Sci USA 2016; 113: 5754-5759.

22. Copps KD, White MF. Regulation of insulin sensitivity by serine/threonine phosphorylation of insulin receptor substrate proteins IRS1 and IRS2. Diabetologia 2012; 55: 2565-2582.

Received December 14, 2016. Accepted December 27, 2016. 